\title{
PROJECTION DECOMPOSITION IN MULTIPLIER ALGEBRAS
}

\author{
VICTOR KAFTAL, P. W. NG, AND SHUANG ZHANG
}

\begin{abstract}
In this paper we present new structural information about the multiplier algebra $\mathcal{N}(\mathcal{A})$ of a $\sigma$-unital purely infinite simple $C^{*}$-algebra $\mathcal{A}$, by characterizing the positive elements $A \in \mathcal{M}(\mathcal{A})$ that are strict sums of projections belonging to $\mathcal{A}$. If $A \notin \mathcal{A}$ and $A$ itself is not a projection, then the necessary and sufficient condition for $A$ to be a strict sum of projections belonging to $\mathcal{A}$ is that $\|A\|>1$ and that the essential norm $\|A\|_{\text {ess }} \geq 1$.

Based on a generalization of the Perera-Rordam weak divisibility of separable simple $C^{*}$-algebras of real rank zero to all $\sigma$-unital simple $C^{*}$-algebras of real rank zero, we show that every positive element of $\mathcal{A}$ with norm greater than 1 can be approximated by finite sums of projections. Based on block tridiagonal approximations, we decompose any positive element $A \in \mathcal{M}(\mathcal{A})$ with $\|A\|>1$ and $\|A\|_{\text {ess }} \geq 1$ into a strictly converging sum of positive elements in $\mathcal{A}$ with norm greater than 1 .
\end{abstract}

\section{Introduction and the main result}

In [9] Fillmore raised the following question: Which positive bounded operators on a separable Hilbert space $\mathcal{H}$ can be written as (finite) sums of projections? Fillmore obtained a characterization of the finite rank operators that are sums of projections (see [9] Theorem 1) and of the bounded operators that are the sums of two projections (see [9] Theorem 2).

For infinite sums of projections with convergence in the strong operator topology, this question arose naturally from work on frame theory by Dykema, Freeman, Kornelson, Larson, Ordower and Weber (see [5]). They proved that a sufficient condition for a positive bounded operator $A \in \mathbb{B}(\mathcal{H})$ to be a (possibly infinite) sum of projections converging in the strong operator topology is that its essential norm $\|A\|_{\text {ess }}$ is greater than 1 (see [5] Theorem 2). This result served as a basis for further work by Kornelson and Larson [13] and then by Antezana, Massey, Ruiz and Stojanoff [1] on decompositions of positive operators into strongly converging sums of rank one positive operators with preassigned norms. In [12], the necessary and sufficient condition for a positive bounded operator to be a strongly converging sum of projections was obtained by the three authors of this article for the $\mathbb{B}(\mathcal{H})$ case and for the case of a countably decomposable type III von Neumann factor, and for the "diagonalizable" case of type II von Neumann factors.

In this paper, we extend the characterization of the positive operators that are sums of projections to the case of bounded module maps (with adjoints defined) on Hilbert $C^{*}$-modules, namely, $\mathbb{B}(\mathcal{H})$ is replaced by the multiplier algebra $\mathcal{M}(\mathcal{A})$ of $\mathcal{A}$. Dealing with multiplier algebras, we replace the strong operator topology by the strict topology. We point out that when $\mathcal{A}$ is reduced to the algebra of complex numbers $\mathbb{C}$, then $\mathbb{B}(\mathcal{H})=\mathcal{M}(\mathcal{K})$, the multiplier algebra of the $C^{*}$-algebra $\mathcal{K}$ of compact operators on a separable Hilbert space, and the ${ }^{*}$-strong operator topology on $\mathbb{B}(\mathcal{H})$ is precisely the strict topology of $\mathcal{M}(\mathcal{K})$. 
In this article we generalize the main result of [5] to certain multiplier algebras, stated as follws.

Theorem 1.1. Let $\mathcal{A}$ be a $\sigma$-unital simple purely infinite $C^{*}$-algebra and $A$ be a positive element of $\mathcal{M}(\mathcal{A})$. Then $A$ is a strictly converging sum of projections belonging to $\mathcal{A}$ if and only if one of the following mutually exclusive conditions hold:

(i) $\|A\|_{\text {ess }}>1$.

(ii) $\|A\|_{\text {ess }}=1$ and $\|A\|>1$.

(iii) $A \in \mathcal{M}(\mathcal{A}) \backslash \mathcal{A}$ is a projection.

(iv) $A$ is the sum of finitely many projections belonging to $\mathcal{A}$.

When $\mathcal{A}$ is unital and hence $\mathcal{M}(\mathcal{A})=\mathcal{A}$, if a positive element $A \in \mathcal{A}$ is a strictly converging sum of (nonzero) projections belonging to $\mathcal{A}$, then the sum must be finite (Proposition 3.1), stated as the case (iv).

The non-trivial case is thus when $A \in \mathcal{M}(\mathcal{A}) \backslash \mathcal{A}$ where $\mathcal{A}$ is $\sigma$-unital but nonunital. Notice that such a $C^{*}$-algebra $(\sigma$-unital but non-unital simple purely infinite) is necessarily stable (see [32] and [22]) and has real rank zero $([29,1.2])$.

The necessity of the conditions (i)-(iii) is given by Corollary 3.3. The sufficiency of (iii) being trivial, the main focus of this paper is to prove the sufficiency of (i) and (ii).

The proof is arranged in the following way.

In section 2 we prove that all non-elementary, $\sigma$-unital, simple $C^{*}$-algebras of real rank zero are weakly divisible in the sense of Perera-Rordam in [19], thus generalizing the previous result of [19] from the separable category to the $\sigma$-unital category. This weak divisibility property and Fillmore's characterization of the finite rank operators in $\mathbb{B}(\mathcal{H})$ enable us to approximate a positive element with a norm greater than 1 by finite sums of projections (Lemma 2.5.)

In section 3 we prove that a positive element $A$ of $\mathcal{M}(\mathcal{A})$ with essential norm $\|A\|_{\text {ess }}>1$ can be written as a strict sum of projections in $\mathcal{A}$.

Section 4 deals with the crucial case when $\|A\|_{\text {ess }}=1$ and $\|A\|>1$. We employ a block tri-diagonal approximation and operator theory techniques to construct a strictly converging sequence of projections $f_{k} \in A$ for which $\left\|f_{k} A f_{k}\right\|>1$ for all $k$ (Lemma 4.4). From that, we decompose $A$ into a strict sum of projections (Proposition 4.6) and conclude the proof.

Aside from the works on the $\mathbb{B}(\mathcal{H})$ and von Neumann factors cases that have been mentioned above, this paper employs some previous results and ideas on the structures of multiplier algebras of simple purely infinite $C^{*}$-algebras scattered in the several papers such as [14], [16], [17], [19], and [21] - [33].

The first and second named authors participated in the NSF supported Workshop in Analysis and Probability, Texas A \& M University, Summer 2006, where they first heard from David Larson about the results in [5] and [13] that stimulated this project.

The first and third author were partially supported by grants from the Charles Phelps Taft Research Center.

\section{Weak divisibility of $\sigma$-unital $C^{*}$-algebras of real rank zero}

In this section we show that in a $\sigma$-unital simple purely infinite $C^{*}$-algebra $\mathcal{A}$, every positive element with norm greater than 1 can be approximated from underneath by finite sums of projections. To do so we first extend to all non-elementary 
$\sigma$-unital $C^{*}$-algebras of real rank zero the property of weak divisibility obtained for separable non-elementary simple $C^{*}$-algebras of real rank zero by Perera and Rordam in $[19,5.3]$. Recall that a $C^{*}$-algebra is called non-elementary if it is neither $\mathcal{K}$ nor $\mathbb{M}_{n}$ for any $n$. A $C^{*}$-algebra is called to be $\sigma$-unital, if it has a strictly positive element $b$, namely, $(b \mathcal{A})^{-}=(\mathcal{A} b)^{-}=\mathcal{A}$. A $C^{*}$-algebra $\mathcal{B}$ is weakly divisible $([19,5.1,5.2])$ if and only if for any nonzero projection $p$ of $\mathcal{B}$ there exists a unital *-homomorphism from $\mathbb{M}_{2} \oplus \mathbb{M}_{3}$ to $p \mathcal{B} p$.

Proposition 2.1. If $\mathcal{A}$ is a non-elementary $\sigma$-unital simple $C^{*}$-algebra of real rank zero, then $\mathcal{A}$ is weakly divisible.

Proof. By [19, Lemma 5.2] it suffices to show that for each nonzero projection $p$ of $\mathcal{A}$ there exists a unital ${ }^{*}$-homomorphism from $\mathbb{M}_{2} \oplus \mathbb{M}_{3}$ into $p \mathcal{A} p$. To prove this, we use the result of divisibility of all projections in any simple $C^{*}$-algebra of real rank zero in $[26,1.1]$ : For each pair of projections $(q, r)$ in $\mathcal{A}$ and each natural number $n$ the projection $q$ can be rewritten as a direct sum of mutually orthogonal subprojections

$$
q=p_{1} \oplus p_{2} \oplus \cdots \oplus p_{2^{n}} \oplus r_{0}
$$

such that $p_{i}$ is equivalent to $p_{j}$ for all pairs $(i, j)$ in the sense of Murray-von Neumann and $r_{0}$ is equivalent to a subprojection of $r$.

Applying this result to the case $q=r=p$ and $n=1$, one has

$$
p=p_{1} \oplus p_{2} \oplus r_{0}
$$

where $p_{1}$ is equivalent to $p_{2}$ and $r_{0}$ is equivalent to a subprojection of $p_{1}$, say $r_{1}$. Choose a partial isometry $v$ such that $p_{1}=v v^{*}, p_{2}=v^{*} v$, and set $r_{2}=v^{*} r_{1} v$. Then

$$
p=\left(p_{1}-r_{1}\right) \oplus\left(p_{2}-r_{2}\right) \oplus r_{0} \oplus r_{1} \oplus r_{2} .
$$

Then $p_{1}-r_{1}$ and $p_{2}-r_{2}$ are equivalent, and so are $r_{0}, r_{1}$ and $r_{2}$. This decomposition of $p$ into these five projections leads to a unital ${ }^{*}$-homomorphism from $\mathbb{M}_{2} \oplus \mathbb{M}_{3}$ into $p \mathcal{A} p$.

The same idea above also proves the following lemma that will be used as one of the technical ingredients in this article.

Lemma 2.2. Let $\mathcal{A}$ be a non-elementary $\sigma$-unital simple $C^{*}$-algebra of real rank zero. Then for every integer $n \geq 1$ and for every nonzero projection $p$ of $\mathcal{A}$ there exists a unital $*$-embedding of $\mathbb{M}_{2^{n}} \oplus \mathbb{M}_{2^{n}+1}$ into $p \mathcal{A} p$.

Proof. Applying $[26,1.1]$ to the case $q=r=p$ and arbitrary natural number $n$, one has

$$
p=p_{1} \oplus p_{2} \oplus \cdots \oplus p_{2^{n}} \oplus r_{0}
$$

where $p_{i}$ is equivalent to $p_{j}$ for all pair $(i, j)$ and $r_{0}$ is equivalent to a subprojection of $p_{1}$, say $r_{1}$. For each $k$ choose a partial isometry $v_{k} \in p \mathcal{A} p$ such that $p_{1}=v_{k} v_{k}^{*}$ and $p_{k}=v_{k}^{*} v_{k}$. Let $r_{k}=v_{k}^{*} r_{1} v_{k}$. Then

$$
p=\left(p_{1}-r_{1}\right) \oplus\left(p_{2}-r_{2}\right) \oplus \cdots \oplus\left(p_{2^{n}}-r_{2^{n}}\right) \oplus r_{0} \oplus r_{1} \oplus r_{2} \oplus \cdots \oplus r_{2^{n}} .
$$

Then $p_{i}-r_{i}$ and $p_{j}-r_{j}$ are equivalent for all pairs $(i, j)$, and so are $r_{0}, r_{1}, \cdots$, $r_{2^{n}}$. This decomposition of $p$ leads to a unital *-homomorphism from $\mathbb{M}_{2^{n}} \oplus \mathbb{M}_{2^{n}+1}$ into $p \mathcal{A} p$.

We need the following approximation property for positive elements in a $C^{*}$ algebra of real rank zero. 
Lemma 2.3. Let $\mathcal{C}$ be a $C^{*}$-algebra of real rank zero and $c$ be any positive element in $\mathcal{C}$. For $\epsilon>0$ there exist pairwise orthogonal projections $p_{1}, p_{2}, \ldots, p_{n}$ in $\mathcal{C}$ and positive real numbers $\alpha_{1}, \alpha_{2}, \ldots, \alpha_{n}$ such that

(i) $\left\|\alpha_{1} p_{1}+\alpha_{2} p_{2}+\ldots+\alpha_{n} p_{n}-c\right\|<\epsilon$

(ii) $\alpha_{1} p_{1}+\alpha_{2} p_{2}+\ldots+\alpha_{n} p_{n} \leq c$.

Proof. Without loss of generality, assume that $\epsilon<2\|c\|$. Let $g$ be the piecewise linear function

$$
g(x)=: \begin{cases}0 & 0 \leq x \leq \epsilon / 2 \\ x-\epsilon / 2 & x>\epsilon / 2\end{cases}
$$

The hereditary subalgebra of $\mathcal{C}$ generated by $\overline{g(c) \mathcal{C} g(c)}$ still has real rank zero ([3]). Thus one can find a positive element $d \in \overline{g(c) \mathcal{C} g(c)}$ with finite spectrum, say $d=\alpha_{1} p_{1}+\alpha_{2} p_{2}+\ldots+\alpha_{n} p_{n}$, such that $\|g(c)-d\|<\epsilon / 2$. It follows that

$$
\left\|c-\alpha_{1} p_{1}+\alpha_{2} p_{2}+\ldots+\alpha_{n} p_{n}\right\| \leq\|c-g(c)\|+\|g(c)-d\|<\epsilon / 2+\epsilon / 2=\epsilon .
$$

The key point is to prove that $d \leq c$. Assume without loss of generality that $\mathcal{C}$ act faithfully and non-degenerately on a Hilbert space $\mathcal{H}$. Let $q=: \chi_{[\epsilon / 2, \infty)}(c)$. For $\xi \in q \mathcal{H}$, one has that

$$
\begin{aligned}
<(c-d) \xi, \xi> & =<(c-g(c)) \xi, \xi>+<(g(c)-d) \xi, \xi> \\
& =\epsilon<\xi, \xi>+<(g(c)-d) \xi, \xi> \\
& \geq(\epsilon-\|g(c)-d\|)<\xi, \xi> \\
& \geq 0 .
\end{aligned}
$$

If $\xi \in q^{\perp} \mathcal{H}$, then $d \xi=0$ because $d \in \overline{g(c) \mathcal{C} g(c)}$, and hence also $<(c-d) \xi, \xi>=<$ $c \xi, \xi>\geq 0$. Therefore, $c \geq d$, as wanted.

We will use the following result due to Fillmore [9, Thm. 1] (see also [5, Prop. $6]$ and $[12,2.5,2.6]$.)

Proposition 2.4. Let tr be the natural (non-normalized) trace on the algebra $\mathbb{M}_{n}$ of $n$ by $n$ complex matrices and $A \in \mathbb{M}_{n}$ be a positive matrix. Then $A$ is a sum of projections in $\mathbb{M}_{n}$ if and only if $\operatorname{tr}(A)$ is an integer and $\operatorname{tr}(A) \geq \operatorname{rank}(A)$.

Recall that all simple purely infinite $C^{*}$-algebras have real rank zero $([29,1.2])$. The following lemma is one of the two central technical ingredients of this article.

Lemma 2.5. Let $\mathcal{A}$ be a $\sigma$-unital purely infinite simple $C^{*}$-algebra and $A \in \mathcal{A}$ be a positive element with $\|A\|>1$. Then for every $\epsilon>0$ there exist positive elements $A_{1}, A_{2} \in \mathcal{A}$ such that

(i) $A=A_{1}+A_{2}$,

(ii) $A_{1}$ is the sum of finitely many projections belonging to $\mathcal{A}$, and

(iii) $\left\|A_{2}\right\|<\epsilon$.

Proof. By Lemma 2.3 we can assume without loss of generality that $A$ is a positive element with finite spectrum and with norm strictly greater than one. Then there are nonzero pairwise orthogonal projections

$$
e_{1}, e_{2}, \ldots, e_{m}, f_{1}, f_{2}, \ldots, f_{n} \in \mathcal{A}
$$


and strictly positive real numbers $\lambda_{1}, \lambda_{2}, \ldots, \lambda_{m}, \mu_{1}, \mu_{2}, \ldots, \mu_{n}$ such that

$$
A=\sum_{i=1}^{m} \lambda_{i} e_{i}+\sum_{j=1}^{n} \mu_{j} f_{j}
$$

where $1<\lambda_{i}$ and $0<\mu_{j} \leq 1$ for $1 \leq i \leq m$ and $1 \leq j \leq n$. Note that $\|A\|>1$ implies $m \geq 1$; but $n=0$ is possible.

Choose $N$ large enough in the form $2^{k}$ such that there are positive integers $k_{i}, k_{i}^{\prime}, l_{j}, l_{j}^{\prime}$ for all $1 \leq i \leq m$ and $1 \leq j \leq n$ satisfying the following inequalities:

$$
\begin{aligned}
& 1<k_{i} / N<\lambda_{i} \text { and } 1<k_{i}^{\prime} /(N+1)<\lambda_{i}, \\
& l_{j} / N<\mu_{j} \text { and } l_{j}^{\prime} /(N+1)<\mu_{j}, \\
& 0<\lambda_{i}-k_{i} / N<\frac{\epsilon}{2} \text { and } 0<\lambda_{i}-k_{i}^{\prime} /(N+1)<\frac{\epsilon}{2}, \\
& 0<\mu_{j}-l_{j} / N<\frac{\epsilon}{2} \text { and } 0<\mu_{j}-l_{j}^{\prime} /(N+1)<\frac{\epsilon}{2} .
\end{aligned}
$$

By Lemma 2.2 there exists for $1 \leq i \leq m$ a unital *-homomorphism from $\mathbb{M}_{N} \oplus$ $\mathbb{M}_{N+1}$ onto a $C^{*}$-subalgebra $\mathcal{B}_{i}$ of the corner $e_{i} \mathcal{A} e_{i}$, and for $1 \leq j \leq n$ there is a unital *-homomorphism from $\mathbb{M}_{N} \oplus \mathbb{M}_{N+1}$ onto a $C^{*}$-subalgebra $\mathcal{C}_{j}$ of $f_{j} \mathcal{A} f_{j}$. Notice that for a given $i$, the projections in $\mathcal{B}_{i}$ that correspond to the minimal projections of $\mathbb{M}_{N}$ are all mutually equivalent, but in general they are not comparable to the minimal projections in $\mathcal{C}_{j}$ or in $\mathcal{B}_{i^{\prime}}$ for $i \neq i^{\prime}$ or to those in $\mathcal{B}_{i}$ that correspond to the minimal projections of $\mathbb{M}_{N+1}$. The identity of $\mathcal{B}_{i}$ is $e_{i}$ and the identity of $\mathcal{C}_{j}$ is $f_{j}$ and this way, each summand $\lambda_{i} e_{i}$ is identified with a direct sum of two diagonal matrices, say $B_{i}=B_{i 1} \oplus B_{i 2}$ in $\mathcal{B}_{i}$, where $B_{i 1}$ is a matrix of size $N \times N, B_{i 2}$ is a matrix of size $(N+1) \times(N+1)$, and both have all diagonal entries $\lambda_{i}$. Similarly, each summand $\mu_{j} f_{j}$ is identified with a direct sum of two diagonal matrices, say $C_{j}=C_{j 1} \oplus C_{j 2}$ in $\mathrm{C}_{j}$, where $C_{j 1}$ is of size $N \times N, C_{j 2}$ is of size $(N+1) \times(N+1)$, and both have all diagonal entries $\mu_{j}$.

Modify $B_{i}=B_{i 1} \oplus B_{i 2}$ to $B_{i}^{\prime}=B_{i 1}^{\prime} \oplus B_{i 2}^{\prime}$ where $B_{i 1}^{\prime}$ has the same matrix units as $B_{i 1}$ but has all diagonal entries $k_{i} / N$ instead of $\lambda_{i}$ and $B_{i 2}^{\prime}$ has the same matrix units as of $B_{i 2}$ but has all diagonal entries $k_{i}^{\prime} /(N+1)$ instead of $\lambda_{i}$. Similarly, modify $C_{j}=C_{j 1} \oplus C_{j 2}$ to $C_{j}^{\prime}=C_{j 1}^{\prime} \oplus C_{j 2}^{\prime}$ by replacing the diagonal entries $\mu_{j}$ of $C_{j 1}$ with $l_{j} / N$ and the diagonal entries $\mu_{j}$ of $C_{j 2}$ with $l_{j}^{\prime} /(N+1)$. Let

$$
A^{\prime}=\sum_{i=1}^{m} B_{i}^{\prime}+\sum_{j=1}^{n} C_{j}^{\prime}
$$

Notice that all the matrices $B_{i 1}^{\prime}$ and $C_{j 1}^{\prime}$ have rank $N$ and all the matrices $B_{i 2}^{\prime}$ and $C_{j 2}^{\prime}$ have rank $N+1$. The conditions defining $k_{j}, k_{j}^{\prime}, l_{j}, l_{j}^{\prime}$ imply:

$$
\begin{aligned}
& 0 \leq A^{\prime} \leq A \text { and }\left\|A-A^{\prime}\right\|<\frac{\epsilon}{2} \\
& \operatorname{tr}\left(B_{i 1}^{\prime}\right)=N\left(k_{i} / N\right)=k_{i} \text { and } \operatorname{tr}\left(B_{i 2}^{\prime}\right)=(N+1) k_{i}^{\prime} /(N+1)=k_{i}^{\prime} . \\
& \operatorname{tr}\left(C_{j 1}^{\prime}\right)=N\left(l_{j} / N\right)=l_{j} \text { and } \operatorname{tr}\left(C_{j 2}^{\prime}\right)=(N+1) l_{j}^{\prime} /(N+1)=l_{j}^{\prime} .
\end{aligned}
$$

Since

$$
\operatorname{tr}\left(B_{i 1}^{\prime}\right)=k_{i}>N=\operatorname{rank}\left(B_{i 1}^{\prime}\right) \text { and } \operatorname{tr}\left(B_{i 2}^{\prime}\right)=k_{i}^{\prime}>N+1=\operatorname{rank}\left(B_{i 2}^{\prime}\right),
$$


by Proposition 2.4 each $B_{i 1}^{\prime}$ and $B_{i 2}^{\prime}$ is a sum of projections. If $n=0, A^{\prime}=$ $\sum_{i=1}^{m} B_{i 1}^{\prime} \oplus B_{i 2}^{\prime}$ is a sum of projections and then setting $A_{1}=A^{\prime}$ and $A_{2}=A-A^{\prime}$ will satisfy the thesis.

From now on assume $n \geq 1$. Since $\sum_{i=2}^{m} B_{i 1}^{\prime} \oplus B_{i 2}^{\prime}$ is a sum of projections, it is enough to prove that $B_{1}^{\prime}+\sum_{j=1}^{n} C_{j}^{\prime}$ is also a sum of projections. Let $e_{11}$ (resp., $e_{12}$ ) be the identity of the copy of $\mathbb{M}_{N}$ (resp., $\mathbb{M}_{N+1}$ ) in $\mathcal{B}_{1}$. Then $B_{1}^{\prime}=\frac{k_{1}}{N} e_{11}+\frac{k_{1}^{\prime}}{N+1} e_{12}$. Since $1<\frac{k_{1}}{N}$, there exists for each $1 \leq j \leq n$ an integer multiple of $N$, say $L_{j}$, such that

$$
L_{j}\left(\frac{k_{1}}{N}-1\right) \geq N-l_{j}
$$

For every $1 \leq j \leq n$, let $f_{j 1}$ be a minimal projection of $C_{j 1}^{\prime}$. Denote by $N \cdot f_{j 1}$ the identity of the copy of $\mathbb{M}_{N}$ in $\mathcal{C}_{j}$. Then $C_{j 1}^{\prime}=\frac{l_{j}}{N}\left(N \cdot f_{j 1}\right)$. Since the corner $e_{11} \mathcal{A} e_{11}$ of $\mathcal{A}$ is still simple and purely infinite, one can recursively find $\sum_{j=1}^{n} L_{j}$ mutually orthogonal projections in $e_{11} \mathcal{A} e_{11}$, where for each $1 \leq j \leq n, L_{j}$ of these projections are equivalent to $f_{j 1}$ and we denote their sum by $L_{j} \cdot f_{j 1}$. Then

$$
D:=\sum_{j=1}^{n} \frac{k_{1}}{N}\left(L_{j} \cdot f_{j 1}\right)+\sum_{j=1}^{n} C_{j 1}^{\prime}=\sum_{j=1}^{n}\left(\frac{k_{1}}{N}\left(L_{j} \cdot f_{j 1}\right)+\frac{l_{j}}{N}\left(N \cdot f_{j 1}\right)\right)
$$

and for each $j, \frac{k_{1}}{N}\left(L_{j} \cdot f_{j 1}\right)+\frac{l_{j}}{N}\left(N \cdot f_{j 1}\right)$ is a matrix of size $L_{j}+N$ and trace $\frac{L_{j} k_{1}}{N}+l_{j} \geq L_{j}+N$ and hence is the sum of projections by Proposition 2.4.

Similarly, there exists for each $1 \leq j \leq n$ an integer multiple of $N+1$, say $L_{j}^{\prime}$, such that

$$
L_{j}^{\prime}\left(\frac{k_{1}}{N+1}-1\right) \geq N+1-l_{j}^{\prime} .
$$

For every $1 \leq j \leq n$, let $f_{j 2}$ be a minimal projection of $C_{j 2}^{\prime},(N+1) \cdot f_{j 2}$ the identity of the copy of $\mathbb{M}_{N+1}$ in $\mathcal{C}_{j} L_{j}^{\prime} \cdot f_{j 2}$ the sum of orthogonal subprojections of $e_{12}$ equivalent to $f_{j 2}$ so that $L_{j}^{\prime} \cdot f_{j 2}$ are also mutually orthogonal. Then

$D^{\prime}:=\sum_{j=1}^{n} \frac{k_{1}}{N+1}\left(L_{j}^{\prime} \cdot f_{j 2}\right)+\sum_{j=1}^{n} C_{j 2}^{\prime}=\sum_{j=1}^{n}\left(\frac{k_{1}}{N+1}\left(L_{j}^{\prime} \cdot f_{j 1}\right)+\frac{l_{j}^{\prime}}{N+1}\left((N+1) \cdot f_{j 1}\right)\right)$

is the sum of projections by the same argument as for $D$.

Finally, let $e^{\prime}=e_{1}-\sum_{j=1}^{n}\left(L_{j} \cdot f_{j 1}\right)-\sum_{j=1}^{n}\left(L_{j}^{\prime} \cdot f_{j}\right)$. Then

$$
B_{1}^{\prime}+\sum_{j=1}^{n} C_{j}^{\prime}=D+D^{\prime}+\frac{k_{1}}{N} e^{\prime}
$$

If $e^{\prime} \neq 0$, by the same argument as for the case $n=0$ one can find a sum of projections $D^{\prime \prime}$ for which $\left\|D^{\prime \prime}-\frac{k_{1}}{N} e^{\prime}\right\|<\frac{\epsilon}{2}$. Then setting

$$
A_{1}:=\sum_{i=2}^{m} B_{i 1}^{\prime} \oplus B_{i 2}^{\prime}+D+D^{\prime}+D^{\prime \prime}
$$

and $A_{2}:=A-A_{1}$ satisfies the thesis. 


\section{The cases $A \in \mathcal{A}$ and $\|A\|_{\text {ess }}>1$}

We first discuss when a positive operator $A$ in a $\sigma$-unital simple purely infinite $C^{*}$-algebra $\mathcal{A}$ is a strict sum of projections in $\mathcal{A}$.

Proposition 3.1. Let $\mathcal{A}$ be a $\sigma$-unital $C^{*}$-algebra with an approximate identity of projections and $A$ be a positive element in $\mathcal{A}$. If $A$ is the strict sum of projections belonging to $\mathcal{A}$, then $A$ must be the sum of finitely many projections belonging to $\mathcal{A}$.

Proof. We will reason by contradiction. Assume that $\left\{p_{k}\right\}_{k=1}^{\infty}$ is an infinite sequence of nonzero projections in $\mathcal{A}$ such that $A=\sum_{k=1}^{\infty} p_{k}$, where the sum converges in the strict topology in $\mathcal{M}(\mathcal{A})$.

Let $\left\{e_{n}\right\}_{n=1}^{\infty}$ be an approximate unit for $\mathcal{A}$ consisting of an increasing sequence of projections. Note that such an increasing approximate identity of projections indeed exists in $\mathcal{A}([28])$. Choose an integer $N \geq 1$ such that for all $n \geq N$, $\left\|A-e_{n} A\right\|<1 / 2$. As a consequence,

$$
\left\|\left(1-e_{N}\right) A\left(1-e_{N}\right)\right\|=\left\|\left(A-e_{N} A\right)\left(1-e_{N}\right)\right\| \leq\left\|A-e_{N} A\right\|<1 / 2
$$

Recall a classical result (for example, see [4, Lemma III.3.1]) that for every $0<\epsilon<1$ there exists a $\delta>0$ such that $p \in \mathcal{A}$ with $\operatorname{dist}\left(p,\left(1-e_{N}\right) \mathcal{A}\left(1-e_{N}\right)\right)<\delta$ implies the existence of a projection $q \in\left(1-e_{N}\right) \mathcal{A}\left(1-e_{N}\right)$ satisfying $\|p-q\|<\epsilon$. Such a projection $q$ is equivalent to $p$ in $\mathcal{A}$. For $\epsilon=1 / 2$ there exists $\delta>0$. Since $\sum_{k=1}^{\infty} p_{k}$ converges in the strict topology on $\mathcal{M}(\mathcal{A})$, let $K \geq 1$ be such that $\left\|p_{k} e_{N}\right\|<\delta / 3$ for all $k \geq K$. Hence, for all $k \geq K$,

$$
\left\|\left(1-e_{N}\right) p_{k}\left(1-e_{N}\right)-p_{k}\right\| \leq\left\|-p_{k} e_{N}-e_{N} p_{k}+e_{N} p_{k} e_{N}\right\| \leq 3\left\|p_{k} e_{N}\right\|<\delta
$$

Thus $\operatorname{dist}\left(p_{K},\left(1-e_{N}\right) \mathcal{A}\left(1-e_{N}\right)\right)<\delta$. It follows from the classical result stated above that there is a projection

$$
q \in\left(1-e_{N}\right) \mathcal{A}\left(1-e_{N}\right) \quad \text { with } \quad\left\|p_{K}-q\right\|<1 / 2 .
$$

Now let $B=\sum_{k \neq K} p_{k}+q$. Then

$$
\|B-A\|=\left\|q-p_{K}\right\|<1 / 2,
$$

and hence,

$$
\left\|\left(1-e_{N}\right) B\left(1-e_{N}\right)-\left(1-e_{N}\right) A\left(1-e_{N}\right)\right\|<1 / 2 .
$$

Applying the triangle inequality, one has

$$
\left\|\left(1-e_{N}\right) B\left(1-e_{N}\right)\right\|<1 / 2+\left\|\left(1-e_{N}\right) A\left(1-e_{N}\right)\right\|<1 .
$$

On the other hand, $\left(1-e_{N}\right) B\left(1-e_{N}\right) \geq q$ implies $\left\|\left(1-e_{N}\right) B\left(1-e_{N}\right)\right\| \geq 1$, a contradiction. Therefore, $A$, as the strict sum of projections, must be a finite sum.

We now turn to handle the sufficient condition $\|A\|_{\text {ess }}>1$. Let us first review some elementary facts about the essential norm, which are formulated only for the special cases that we will work with. Let $\mathcal{A}$ be a non-unital $C^{*}$-algebra, let $\pi$ be the canonical homomorphism from $\mathcal{M}(\mathcal{A})$ onto the corona algebra $\mathcal{M}(\mathcal{A}) / \mathcal{A}$, and for every $A \in \mathcal{M}(\mathcal{A})$, let $\|A\|_{\text {ess }}:=\|\pi(A)\|$ denote the essential norm.

Lemma 3.2. Let $\mathcal{A}$ be a non-unital $C^{*}$-algebra.

(i) For every positive $A \in \mathcal{M}(\mathcal{A})$,

$$
\|A\|_{\text {ess }}=\inf \left\{\|A(I-a)\| \mid a \in \mathcal{A}^{+},\|a\| \leq 1\right\} .
$$


(ii) Let $A \in \mathcal{M}(\mathcal{A}) \backslash \mathcal{A}$ be a positive element, and let $a_{n}$ be a monotone increasing sequence of positive elements of $\mathcal{A}$ converging to $A$ in the strict topology. Then

$$
\|A\|_{\text {ess }}=\inf _{n}\left\|A-a_{n}\right\| \text {. }
$$

Proof.

(i) Since $A a \in \mathcal{A}$ for every $a \in \mathcal{A}$, it follows that $\|A\|_{\text {ess }}=\|A(I-a)\|_{\text {ess }} \leq\|A(I-a)\|$ and hence

$$
\|A\|_{\text {ess }} \leq \inf \left\{\|A(I-a)\| \mid a \in \mathcal{A}^{+},\|a\| \leq 1\right\} .
$$

If $\|A\|_{\text {ess }}=\|A\|$, then the reverse inequality holds by choosing $a=0$, so assume that $\|A\|>\|A\|_{\text {ess }}$. Let $0<\epsilon<\|A\|-\|A\|_{\text {ess }}$, let $h$ be the positive continuous function on the interval $[0,\|A\|]$ defined as

$$
h(t):= \begin{cases}0 & t \in\left[0,\|A\|_{\text {ess }}\right] \\ \text { linear } & t \in\left[\|A\|_{\text {ess }},\|A\|_{\text {ess }}+\epsilon\right], \\ 1 & t \in\left[\|A\|_{\text {ess }}+\epsilon,\|A\|\right]\end{cases}
$$

and let $a:=h(A)$. Clearly, $a \geq 0$ and $\|a\|=1$. Via the Gelfand's transformation, identify $C^{*}(\pi(A))$ with the algebra of complex-valued continuous functions $C\left(\sigma_{e}(A)\right)$ defined on the essential spectrum $\sigma_{e}(A)$ of $A$. Since $h$ vanishes on $\sigma_{e}(A)$ and $h \circ \pi=\pi \circ h$, it follows that $\pi(h(A))=0$ and hence $h(A) \in \mathcal{A}$. Moreover,

$$
\|A(I-a)\|=\|t(1-h(t))\|_{\infty} \leq\|A\|_{\text {ess }}+\epsilon,
$$

whence

$$
\inf \left\{\|A(I-a)\| \mid a \in \mathcal{A}^{+},\|a\| \leq 1\right\} \leq\|A\|_{\text {ess }} .
$$

Thus equality holds, proving (i).

(ii) Since for every $n$

$$
\|A\|_{\text {ess }}=\left\|A-a_{n}\right\|_{\text {ess }} \leq\left\|A-a_{n}\right\|
$$

it follows that

$$
\|A\|_{e s s} \leq \inf _{n}\left\|A-a_{n}\right\| .
$$

For every positive contraction $a \in \mathcal{A}$ and every $n$

$$
\left\|A-a_{n}\right\|^{1 / 2}=\left\|\left(A-a_{n}\right)^{1 / 2}\right\| \leq\left\|\left(A-a_{n}\right)^{1 / 2} a\right\|+\left\|\left(A-a_{n}\right)^{1 / 2}(I-a)\right\| .
$$

Since $0 \leq A-a_{n} \leq A$,

$$
\left\|\left(A-a_{n}\right)^{1 / 2}(I-a)\right\|^{2}=\left\|(I-a)\left(A-a_{n}\right)(I-a)\right\| \leq\|(I-a) A(I-a)\|=\left\|A^{1 / 2}(I-a)\right\|^{2} .
$$

But then

$$
\left\|A^{1 / 2}(I-a)\right\| \geq\left\|\left(A-a_{n}\right)^{1 / 2}(I-a)\right\| \geq\left\|A-a_{n}\right\|^{1 / 2}-\left\|\left(A-a_{n}\right)^{1 / 2} a\right\| .
$$

Since $A-a_{n} \rightarrow 0$ in the strict topology it follows that $\left\|\left(A-a_{n}\right)^{1 / 2} a\right\| \rightarrow 0$. Since $a_{n}$ is monotone increasing, it follows that $\left\|A-a_{n}\right\|^{1 / 2} \rightarrow \inf \left\|A-a_{n}\right\|^{1 / 2}$ and hence

$$
\left\|A^{1 / 2}(I-a)\right\| \geq \inf _{n}\left\|A-a_{n}\right\|^{1 / 2}
$$

Thus

$$
\inf \left\{\left\|A^{1 / 2}(I-a)\right\| \mid a \in \mathcal{A}^{+},\|a\| \leq 1\right\} \geq \inf _{n}\left\|A-a_{n}\right\|^{1 / 2}
$$

and by (i),

$$
\left\|A^{1 / 2}\right\|_{e s s} \geq \inf _{n}\left\|A-a_{n}\right\|^{1 / 2}
$$


Since $\|A\|_{\text {ess }}=\left\|A^{1 / 2}\right\|_{\text {ess }}^{2}$, it follows that

$$
\|A\|_{\text {ess }} \geq \inf _{n}\left\|A-a_{n}\right\|
$$

which concludes the proof.

Corollary 3.3. Let $\mathcal{A}$ be a non-unital $C^{*}$-algebra and let $A=\sum_{j=i}^{\infty} a_{j}$ where $a_{j} \in \mathcal{A}^{+},\left\|a_{j}\right\| \geq 1$ for all $j$ and the series converges in the strict topology of $\mathcal{M}(\mathcal{A})$. Then $\|A\|_{\text {ess }} \geq 1$.

Every $\sigma$-unital $C^{*}$-algebra $\mathcal{A}$ has a strictly positive element $b \in \mathcal{A}$, i.e., a positive element for which $(b \mathcal{A})^{-}=(\mathcal{A} b)^{-}=\mathcal{A}$. As usual, one can assume that $\|b\|=1$. Define a seminorm on $\mathcal{M}(\mathcal{A})$, say $\|\cdot\|_{b}$, by

$$
\|m\|_{b}:=\|m b\|+\|b m\| \text { for all } m \in \mathcal{M}(\mathcal{A}) .
$$

Clearly, $\|\cdot\|_{b}$ generates the strict topology on $\mathcal{M}(\mathcal{A})$. Note that $\|m\|_{b} \leq 2\|m\|$ for all $m \in \mathcal{M}(\mathcal{A})$.

Proposition 3.4. Let $\mathcal{A}$ be a $\sigma$-unital non-unital purely infinite simple $C^{*}$-algebra and let $A \in \mathcal{M}(\mathcal{A})$ be a positive element with $\|A\|_{\text {ess }}>1$. Then $A$ is a strict sum of projections.

Proof. Every $\sigma$-unital, non-unital $C^{*}$-algebra of real rank zero has an approximate identity of projections; such an approximate identity can always be chosen to be countable and increasing, say $\left\{e_{j}\right\}([28])$.

Let $q_{j}=e_{j}-e_{j-1}$ setting $e_{o}=0$. Then $\sum_{j=1}^{\infty} q_{j}=I$, where the convergence is in the strict topology. Furthermore,

$$
A=\sum_{j=1}^{\infty} A^{1 / 2} q_{j} A^{1 / 2}
$$

where the convergence is also in the strict topology. By Lemma 3.2 (ii),

$$
\left\|\sum_{j=n}^{\infty} A^{1 / 2} q_{j} A^{1 / 2}\right\| \geq\|A\|_{e s s}
$$

for every $n$. Thus the condition $\|A\|_{\text {ess }}>1$ allows us to find a strictly increasing sequence of integers $n_{k}$ starting with $n_{0}=1$ such that

$$
\left\|\sum_{j=n_{k-1}}^{n_{k}-1} A^{1 / 2} q_{k} A^{1 / 2}\right\|>1
$$

for every $k$. Let

$$
a_{k}:=\sum_{j=n_{k-1}}^{n_{k}-1} A^{1 / 2} q_{k} A^{1 / 2} .
$$

Then $a_{k}$ is a positive element in $\mathcal{A}^{+}$with $\left\|a_{k}\right\|>1$ for every $k$ and $A=\sum_{k=1}^{\infty} a_{k}$ in the strict topology. Thus

$$
\left\|\sum_{k=n}^{\infty} a_{k}\right\|_{b} \rightarrow 0
$$

Apply Lemma 2.5 to $a_{1}$ to obtain a finite sum of projections $d_{1} \in \mathcal{A}, d_{1} \leq a_{1}$ with

$$
|| d_{1}-a_{1} \mid<\frac{1}{2}\left\|\sum_{k=2}^{\infty} a_{k}\right\|_{b} .
$$


Let $b_{1}:=a_{1}-d_{1} \in \mathcal{A}^{+}$and hence $\left\|b_{1}\right\|_{b} \leq\left\|\sum_{k=2}^{\infty} a_{k}\right\|_{b}$. Then $A-d_{1}=b_{1}+\sum_{k=2}^{\infty} a_{k}$, and hence,

$$
\left\|A-d_{1}\right\|_{b} \leq\left\|b_{1}\right\|_{b}+\left\|\sum_{k=2}^{\infty} a_{k}\right\|_{b} \leq 2\left\|\sum_{k=2}^{\infty} a_{k}\right\|_{b} .
$$

Next, since $b_{1}+a_{2} \in \mathcal{A}^{+}$and $\left\|b_{1}+a_{2}\right\| \geq\left\|a_{2}\right\|>1$, we can apply Lemma 2.5 to $b_{1}+a_{2}$ to obtain a finite sum of projections $d_{2} \leq b_{1}+a_{2}$ with

$$
\left\|b_{1}+a_{2}-d_{2}\right\| \leq \frac{1}{2}\left\|\sum_{k=3}^{\infty} a_{k}\right\|_{b}
$$

Thus, iterating, we can find for each $k$ a finite sum $d_{k}$ of projections in $\mathcal{A}$ so that

$$
\left\|A-\sum_{k=1}^{n} d_{k}\right\| \leq 2\left\|\sum_{k=n+1}^{\infty} a_{k}\right\|_{b} \rightarrow 0 .
$$

This proves that the sum $\sum_{k=1}^{\infty} d_{k}$ converges to $A$ in the strict topology, and hence that $A$ is a strict sum of projections, as claimed.

Remark 3.5. In the course of the above proof we have proven that if $\mathcal{A}$ is a $\sigma$ unital non-unital purely infinite simple $C^{*}$-algebra, and $A=\sum_{k=1}^{\infty} a_{k}$ in the strict topology, where $a_{k} \in \mathcal{A}^{+}$and $\left\|a_{k}\right\|>1$ for all $k$, then $A$ is a strict sum of projections. The condition "purely infinite and simple" is the key assumption for the conclusion to hold in the eyes of key Lemma 2.5.

\section{The case $\|A\|_{\text {ess }}=1$ and $\|A\|>1$}

The objective of this section is to prove that $\|A\|_{\text {ess }}=1$ and $\|A\|>1$ suffice to have $A$ written as a strictly converging sum of projections in $\mathcal{A}$. We start with some technical preparations.

Lemma 4.1. Let $\mathcal{A}$ be any $C^{*}$-algebra of real rank zero and $A$ be a positive element in $\mathcal{M}(\mathcal{A})$ such that $\|A\|_{\text {ess }}=1$ and $\|A\|>1$. Then there exist a positive element $A^{\prime} \in \mathcal{M}(\mathcal{A})$, a real number $\lambda>1$, and a nonzero projection $p \in \mathcal{A}$ such that

(i) $\left\|A^{\prime}\right\|_{\text {ess }}=1$,

(ii) $A^{\prime} p=p A^{\prime}=0$,

(iii) $A^{\prime}+\lambda p \leq A$.

Proof. Let $\delta=\|A\|-1$. Define two positive continuous functions $h_{1}(t)$ and $h_{2}(t)$ on $[0,\|A\|]$ as follows:

$$
h_{1}(t):=\left\{\begin{array}{ll}
0 & t \in\left[0,1+\frac{\delta}{2}\right] \\
\text { linear } & t \in\left[1+\frac{\delta}{2}, 1+\frac{3 \delta}{4}\right] \\
t & t \in\left[1+\frac{3 \delta}{4},\|A\|\right]
\end{array} \quad \text { and } \quad h_{2}(t):= \begin{cases}t & t \in\left[0,1+\frac{\delta}{4}\right] \\
\text { linear } & t \in\left[1+\frac{\delta}{4}, 1+\frac{\delta}{2}\right] \\
0 & t \in\left[1+\frac{\delta}{2},\|A\|\right]\end{cases}\right.
$$

Clearly, $h_{1}(t)+h_{2}(t) \leq t$ and $h_{1}(t) h_{2}(t)=0$ for all $t$, hence, $h_{1}(A)+h_{2}(A) \leq A$ and $h_{1}(A) h_{2}(A)=0$. Let $\pi$ be the quotient map from $\mathcal{M}(\mathcal{A})$ to the corona algebra $\mathcal{M}(\mathcal{A}) / \mathcal{A}$. Reasoning as in Lemma 3.2, $h_{1}(A) \in \mathcal{A}$ and $\left\|h_{2}(A)\right\|_{\text {ess }}=\|A\|_{\text {ess }}=1$. Applying Lemma 2.3, approximate $h_{1}(A)$ by a positive element of finite spectrum satisfying

$$
\alpha_{1} p_{1}+\alpha_{2} p_{2}+\cdots+\alpha_{m} p_{m} \leq h_{1}(A)
$$

where $p_{i}$ are pairwise orthogonal nonzero projections in $\mathcal{A}$. For a sufficient approximation, $\alpha_{i}>1$ holds for at least one $i_{0}$. Set $\lambda:=\alpha_{i_{0}}, p:=p_{i_{0}}$, and $A^{\prime}=h_{2}(A)$. 
Then (i) is satisfied. Since $\lambda p \leq h_{1}(A)$ and hence $A^{\prime}+\lambda p \leq A$, i.e., (iii) is satisfied. Since $h_{1}(A) A^{\prime}=A^{\prime} h_{1}(A)=0$, it follows that $A^{\prime} p=p A^{\prime}=0$. i.e., (ii) is satisfied.

The content of the following lemma can be found in the proof of Theorem 2.2 of [25].

Lemma 4.2. Let $\mathcal{A}$ be a $\sigma$-unital, non-unital $C^{*}$-algebra that has an approximate identity of projections. If $A \in \mathcal{M}(\mathcal{A})$ is a positive element, then for every $\epsilon>0$ there exist three positive elements $A_{1}, A_{2}, A_{3} \in \mathcal{M}(\mathcal{A})$ and there is a self-adjoint element $a \in \mathcal{A}$ with $\|a\|<\epsilon$ such that

$$
A=A_{1}+A_{2}+A_{3}+a,
$$

where all $A_{1}, A_{2}, A_{3}$ are in block-diagonal forms (see the detailed descriptions in the following proof).

Proof. The details were given in the proof of [25, 2.2], but we sketch them here for the convenience of the readers. Let $\left\{e_{j}\right\}$ be an approximate unit of projections. For $i \geq 1$ we will view $\left(e_{i}-e_{i-1}\right) A^{\frac{1}{2}}\left(e_{j}-e_{j-1}\right)$ as the $(i, j)$-entry of $A^{\frac{1}{2}}$, and view $\left(e_{n_{i+1}}-e_{n_{i}}\right) A^{\frac{1}{2}}\left(e_{n_{j}}-e_{n_{j-1}}\right)$ as the $(i, j)$-block entry of $A^{\frac{1}{2}}$.

Using a standard argument recursively on $A^{\frac{1}{2}}$ one can find an increasing sequence of indices $\left\{n_{i}\right\}$ starting with $n_{0}=0$ such that $A^{\frac{1}{2}}$ can be rewritten as a sum $A^{\frac{1}{2}}=: X+a$ of two self-adjoint elements, where setting $e_{n_{0}}=0$,

$$
a=: \sum_{i=1}^{\infty}\left\{\left(e_{n_{i}}-e_{n_{i-1}}\right) A^{\frac{1}{2}}\left(1-e_{n_{i+1}}\right)+\left(1-e_{n_{i+1}}\right) A^{\frac{1}{2}}\left(e_{n_{i}}-e_{n_{i-1}}\right)\right\}
$$

satisfies $\|a\| \leq \frac{\epsilon}{2 \sqrt{\|A\|}+1}$ and $X=: A^{\frac{1}{2}}-a$. Then

$$
\begin{aligned}
X & =\sum_{i=1}^{\infty}\left(e_{n_{i+1}}-e_{n_{i}}\right) A^{\frac{1}{2}}\left(e_{n_{i}}-e_{n_{i-1}}\right) \\
& +\sum_{i=1}^{\infty}\left(e_{n_{i}}-e_{n_{i-1}}\right) A^{\frac{1}{2}}\left(e_{n_{i}}-e_{n_{i-1}}\right) \\
& +\sum_{i=1}^{\infty}\left(e_{n_{i}}-e_{n_{i-1}}\right) A^{\frac{1}{2}}\left(e_{n_{i+1}}-e_{n_{i}}\right),
\end{aligned}
$$

the second sum above can be viewed as the main block diagonal, the first sum (resp., last sum) can be viewed as the first block diagonal below (resp., above) the main one. In this way, $X$ is said to have a tri-block diagonal form.

Define

$$
\begin{aligned}
& A_{1}=: X \sum_{i=1}^{\infty}\left(e_{n_{3 i-2}}-e_{n_{3 i-3}}\right) X, \\
& A_{2}=: X \sum_{i=1}^{\infty}\left(e_{n_{3 i-1}}-e_{n_{3 i-2}}\right) X, \\
& A_{3}=: X \sum_{i=1}^{\infty}\left(e_{n_{3 i}}-e_{n_{3 i-1}}\right) X .
\end{aligned}
$$


Clearly, $A_{1}+A_{2}+A_{3}=X^{2}=A-A^{\frac{1}{2}} a_{0}-a_{0} A^{\frac{1}{2}}+a_{0}^{2}$ and all three sums $A_{1}, A_{2}$, and $A_{3}$ strictly converge to positive elements of $\mathcal{M}(\mathcal{A})$. Set $a=A^{\frac{1}{2}} a_{0}+a_{0} A^{\frac{1}{2}}-a_{0}^{2}$. Then $\|a\|<\epsilon$.

Via multiplication one sees that $A_{1}$ is of block diagonal with respect to the decomposition of the identity (of $\mathcal{M}(\mathcal{A})$ )

$$
1=e_{n_{3}} \oplus\left(e_{n_{6}}-e_{n_{3}}\right) \oplus \cdots \oplus\left(e_{n_{3 i}}-e_{\left.n_{3 i-3}\right)}\right) \oplus \cdots,
$$

$A_{2}$ is of block diagonal with respect to the decomposition

$$
1=\left(e_{n_{4}}-e_{n_{1}}\right) \oplus\left(e_{n_{7}}-e_{n_{4}}\right) \oplus \cdots \oplus\left(e_{n_{3 i+1}}-e_{n_{3 i-2}}\right) \oplus \cdots,
$$

and $A_{3}$ is of block diagonal with respect to the decomposition

$$
1=\left(e_{n_{5}}-e_{n_{2}}\right) \oplus\left(e_{n_{8}}-e_{n_{4}}\right) \oplus \cdots \oplus\left(e_{n_{3 i+2}}-e_{n_{3 i-1}}\right) \oplus \cdots .
$$

Lemma 4.3. Let $\mathcal{A}$ be a $\sigma$-unital, non-unital $C^{*}$-algebra of real rank zero and let $A \in \mathcal{M}(\mathcal{A}) \backslash \mathcal{A}$ be a positive element. Then for every $\epsilon>0$ there exist a sequence $\left\{q_{k}\right\}_{k=1}^{\infty}$ of pairwise orthogonal nonzero projections in $\mathcal{A}$, a bounded sequence $\left\{\lambda_{k}\right\}_{k=1}^{\infty}$ of positive real numbers, a positive element $A_{0} \in \mathcal{M}(\mathcal{A})$, and a self-adjoint element $a \in \mathcal{A}$ such that the following hold:

(i) $\|a\|<\epsilon$.

(ii) $\sum_{k=1}^{\infty} q_{k}$ converges in the strict topology of $\mathcal{M}(\mathcal{A})$.

(iii) $A=A_{0}+\sum_{k=1}^{\infty} \lambda_{k} q_{k}+a$.

(iv) $\lim _{k \rightarrow \infty} \lambda_{k}=\|A\|_{\text {ess }}$.

Proof. Applying Lemma 4.2, one has a decomposition

$$
A=A_{1}+A_{2}+A_{3}+a
$$

where $a \in \mathcal{A}$ is self-adjoint, $\|a\|<\epsilon, A_{1}, A_{2}, A_{3} \in \mathcal{M}(\mathcal{A})$ are positive element in block-diagonal forms, as described in the proof of 4.2. Let $a_{i, j}$ be the jth-block on the diagonal of $A_{i}$ for $i=1,2,3$ and $j=1,2, \cdots$. All $a_{i, j}$ are positive elements in $\mathcal{A}$ and $\sum_{i=1}^{3} \sum_{j=1}^{\infty} a_{i, j}=A_{1}+A_{2}+A_{3}$ converges in the strict topology.

We construct by induction a sequence of positive numbers $\lambda_{k}$ and mutually orthogonal projections $q_{k} \in \mathcal{A}$ such that for each $k$

$$
\begin{gathered}
\|A\|_{\text {ess }} \geq \lambda_{k}>\|A\|_{\text {ess }}-1 / 2^{k} \\
\sum_{j=1}^{k} \lambda_{k} q_{k} \leq \sum_{i=1}^{3} \sum_{j=1}^{n_{k}} a_{i, j},
\end{gathered}
$$

where $\left\{n_{k}\right\}$ in an increasing sequence of natural numbers.

For $k=1$, since

$$
\left\|\sum_{i=1}^{3} \sum_{j=1}^{n} a_{i, j}\right\| \uparrow\|A-a\| \geq\|A-a\|_{e s s}=\|A\|_{e s s},
$$

we can choose an integer $n_{1}$ such that $\left\|\sum_{i=1}^{3} \sum_{j=1}^{n_{1}} a_{i, j}\right\|>\|A\|_{\text {ess }}-1 / 2$. By Lemma 2.3 applied to $\sum_{i=1}^{3} \sum_{j=1}^{n_{1}} a_{i, j} \in \mathcal{A}$ one can find an approximation of $\sum_{i=1}^{3} \sum_{j=1}^{n_{1}} a_{i, j}$ by a positive element of finite spectrum belonging to $\mathcal{A}, \sum_{i=1}^{m} \alpha_{i} p_{i}$, with $\alpha_{i}>0, p_{i}$ 
mutually orthogonal nonzero projections of $\mathcal{A}$, and

$$
\begin{aligned}
& \sum_{i=1}^{m} \alpha_{i} p_{i} \leq \sum_{i=1}^{3} \sum_{j=1}^{n_{1}} a_{i, j}, \\
& \left\|\sum_{i=1}^{3} \sum_{j=1}^{n_{1}} a_{i, j}-\sum_{i=1}^{m} \alpha_{i} p_{i}\right\| \leq \frac{1}{2}\left(\|A\|_{\text {ess }}-1 / 2\right) \| .
\end{aligned}
$$

Then at least one of $\alpha_{1}, \alpha_{2}, \cdots, \alpha_{m}$, say $\alpha_{j}$, satisfies

$$
\alpha_{j}>\|A\|_{\text {ess }}-1 / 2 \text {. }
$$

Let $\lambda_{1}=: \min \left\{\alpha_{j},\|A\|_{\text {ess }}\right\}$ and $q_{1}=: p_{j}$, where $q_{1}$ is a nonzero projection from $\mathcal{A}$. Then one has the desired inequality:

$$
\lambda_{1} q_{1} \leq \sum_{i=1}^{3} \sum_{j=1}^{n_{1}} a_{i, j}
$$

For $k=2$ take an integer $m \geq n_{1}+3$ so that the sum $\sum_{i=1}^{3} \sum_{j=m+1}^{\infty} a_{i, j}$ is orthogonal to $\sum_{i=1}^{3} \sum_{j=1}^{n_{1}} a_{i, j}$ in the sense

$$
\left(\sum_{i=1}^{3} \sum_{j=m+1}^{\infty} a_{i, j}\right)\left(\sum_{i=1}^{3} \sum_{j=1}^{n_{1}} a_{i, j}\right)=\left(\sum_{i=1}^{3} \sum_{j=1}^{n_{1}} a_{i, j}\right)\left(\sum_{i=1}^{3} \sum_{j=m+1}^{\infty} a_{i, j}\right)=0 .
$$

Such an $m$ exists by the construction of $A_{1}, A_{2}, A_{3}$.

Since $\left\|\sum_{i=1}^{3} \sum_{j=m+1}^{n} a_{i, j}\right\|$ increases to $\left\|A-a-\sum_{i=1}^{3} \sum_{j=1}^{m} a_{i, j}\right\|$ and

$$
\left\|A-a-\sum_{i=1}^{3} \sum_{j=1}^{m} a_{i, j}\right\| \geq\left\|A-a-\sum_{i=1}^{3} \sum_{j=1}^{m} a_{i, j}\right\|_{\text {ess }}=\|A\|_{\text {ess }},
$$

repeating the above argument for $k=1$, choose an integer $n_{2}>m$ such that

$$
\left\|\sum_{i=1}^{3} \sum_{j=m+1}^{n_{2}} a_{i, j}\right\|>\|A\|_{\text {ess }}-1 / 2^{2} .
$$

As for the case $k=1$, one can choose a nonzero projection $q_{2}$ in $\mathcal{A}$ and $\lambda_{2}>0$ such that

$$
\begin{aligned}
& \|A\|_{\text {ess }} \geq \lambda_{2}>\|A\|_{\text {ess }}-1 / 2^{2}, \\
& \lambda_{2} q_{2} \leq \sum_{i=1}^{3} \sum_{j=m+1}^{n_{2}} a_{i, j} .
\end{aligned}
$$

Clearly, $q_{2} q_{1}=0$ and the obvious inequality

$$
\sum_{i=1}^{3} \sum_{j=m+1}^{n_{2}} a_{i, j} \leq \sum_{i=1}^{3} \sum_{j=n_{1}+1}^{n_{2}} a_{i, j}
$$

guarantees that

$$
\lambda_{1} q_{1}+\lambda_{2} q_{2} \leq \sum_{i=1}^{3} \sum_{j=1}^{n_{2}} a_{i, j} .
$$

Proceeding recursively, one constructs a sequence of pairwise orthogonal projections $\left\{q_{k}\right\}$ and a sequence of positive numbers $\left\{\lambda_{k}\right\}$ with the required properties. 
It is now routine to prove that $\sum_{k=1}^{\infty} \lambda_{k} q_{k}$ and hence also $\sum_{k=1}^{\infty} q_{k}$ converge in the strict topology of $\mathcal{M}(\mathcal{A})$. Then setting

$$
A_{o}=A_{1}+A_{2}+A_{3}-\sum_{k=1}^{\infty} \lambda_{k} q_{k} \in \mathcal{M}(\mathcal{A})
$$

satisfies (i)-(iv).

We now reach our second key lemma.

Lemma 4.4. Let $\mathcal{A}$ be a $\sigma$-unital non-unital purely infinite simple $C^{*}$-algebra and $A$ be a positive element of $\mathcal{M}(\mathcal{A})$ such that $\|A\|_{\text {ess }}=1$ and $\|A\|>1$. Then there exists a sequence $\left\{f_{k}\right\}_{k=1}^{\infty}$ of pairwise orthogonal projections in $\mathcal{A}$ such that

(i) $\sum_{k=1}^{\infty} f_{k}$ converges in the strict topology in $\mathcal{M}(\mathcal{A})$, and

(ii) $\left\|f_{k} A f_{k}\right\|>1$ for all $k$.

Proof. By Lemma 4.1 there is a positive element $A^{\prime}$ of $\mathcal{M}(\mathcal{A})$ with $\left\|A^{\prime}\right\|_{\text {ess }}=1$, a projection $q_{0}$ of $\mathcal{A}$ with $q_{0} A^{\prime}=A^{\prime} q_{0}=0$, and a scalar $\lambda_{0}>1$ such that

$$
A_{0}:=A-A^{\prime}-\lambda_{0} q_{0} \geq 0 .
$$

By Lemma 4.3 applied to $A^{\prime}$ and $\epsilon=1$, there is a sequence of positive real numbers $\lambda_{k}^{\prime} \rightarrow 1$, a sequence of pairwise orthogonal nonzero projections $\left\{q_{k}^{\prime}\right\}_{k=1}^{\infty} \in$ $\mathcal{A}$, a self adjoint element $a=a^{*} \in \mathcal{A}$, and a positive element $A_{0}^{\prime} \in \mathcal{M}(\mathcal{A})$ such that

$$
A^{\prime}=A_{0}^{\prime}+\sum_{k=1}^{\infty} \lambda_{k}^{\prime} q_{k}^{\prime}+a \text {. }
$$

Notice that we can choose $q_{0} q_{k}^{\prime}=q_{k}^{\prime} q_{0}=0$ for all $k$ because $q_{0} A^{\prime}=A^{\prime} q_{0}=0$ (just replace $\mathcal{A}$ with $\left(1-q_{0}\right) \mathcal{A}\left(1-q_{0}\right)$ when applying Lemma 4.3).

Choose a subsequence $\lambda_{k}:=\lambda_{n_{k}}^{\prime}$ such that

$$
\left|\lambda_{k}-1\right|<\left(\lambda_{0}-1\right) / 4^{k+1} \text {. }
$$

Since $\sum_{k \geq 1} q_{n_{k}}^{\prime}$ still converges in the strict topology of $\mathcal{M}(\mathcal{A})$, by passing if necessary to a subsequence, one can further assume that

$$
\max \left\{\left\|\left(a_{+}\right)^{1 / 2} q_{n_{k}}^{\prime}\right\|,\left\|\left(a_{-}\right)^{1 / 2} q_{n_{k}}^{\prime}\right\|\right\}<\frac{\sqrt{\lambda_{0}-1}(\sqrt{2}-1)}{2^{k+1} \sqrt{2}},
$$

where $a_{-}$and $a_{+}$denote the negative and positive parts of $a$, respectively. Since $\mathcal{A}$ is simple and purely infinite, for each $k \geq 1$ there exists a subprojection $q_{k}$ of $q_{n_{k}}^{\prime}$ such that $q_{k} \sim q_{0}$.

Set

$$
\begin{aligned}
q & :=\sum_{k=0}^{\infty} q_{k} \\
A_{00} & =A_{0}+A_{0}^{\prime}+\sum_{i \notin\left\{n_{k}\right\}} \lambda_{i} q_{i}^{\prime}+\sum_{k=1}^{\infty} \lambda_{n_{k}}^{\prime}\left(q_{n_{k}}^{\prime}-q_{k}\right) .
\end{aligned}
$$

Then $q$ is a projection of $\mathcal{M}(\mathcal{A})$ (as a direct sum of countably many mutually orthogonal copies of $\left.q_{0}\right), A_{00}$ is a positive element of $\mathcal{M}(\mathcal{A})$, and

$$
A=A_{00}+\sum_{k=0}^{\infty} \lambda_{k} q_{k}+a
$$


Let $\left\{e_{i}\right\}_{i=0}^{\infty}$ be the sequence of mutually orthogonal rank-one projections in $\mathbb{B}\left(l_{2}\right)$ corresponding to the standard basis of $\ell_{2}$ and let $\rho$ be a unital (isometrical) *embedding $\mathbb{B}\left(l_{2}\right) \rightarrow q \mathcal{N}(\mathcal{A}) q$ for which

$$
\rho\left(e_{i}\right)=q_{i} \text { for all } i \geq 0 .
$$

It is easy to verify that the following matrix $u$ is unitary

$$
\left(\begin{array}{ccccccccc}
1 / \sqrt{2} & -1 / \sqrt{2} & 0 & \ldots & & & & & \\
(1 / \sqrt{2})^{2} & (1 / \sqrt{2})^{2} & -1 / \sqrt{2} & 0 & \ldots & & & \\
\ldots \ldots & & & & & & & \\
\ldots \ldots & & & & & & & \\
(1 / \sqrt{2})^{n} & (1 / \sqrt{2})^{n} & (1 / \sqrt{2})^{n-1} & \ldots & (1 / \sqrt{2})^{3} & (1 / \sqrt{2})^{2} & -1 / \sqrt{2} & 0 & \ldots \\
\ldots & & & & & & & &
\end{array}\right)
$$

and thus $\rho(u)$ is a unitary element of $q \mathcal{N}(\mathcal{A}) q$. Define for all $k \geq 0$

$$
f_{k}=: \rho\left(u^{*} e_{k} u\right) .
$$

Then $\left\{f_{k}\right\}_{k=0}^{\infty}$ is a sequence of pairwise orthogonal (equivalent) projections in $\mathcal{A}$ and

$$
\sum_{k=0}^{\infty} f_{k}=\rho(u)\left(\sum_{k=0}^{\infty} q_{k}\right) \rho(u)^{*}=q .
$$

Since $A_{00} \geq 0$ it follows from (3) that

$$
\begin{aligned}
\left\|f_{k} A f_{k}\right\| & \geq\left\|f_{k}\left(\sum_{j=0}^{\infty} \lambda_{j} q_{j}\right) f_{k}\right\|-\left\|f_{k} a f_{k}\right\| \\
& =\left\|\rho\left(u^{*} e_{k} u\left(\sum_{j=0}^{\infty} \lambda_{j} e_{j}\right) u^{*} e_{k} u\right)\right\|-\left\|f_{k} a f_{k}\right\| \\
& =\left\|e_{k} u\left(\sum_{j=0}^{\infty} \lambda_{j} e_{j}\right) u^{*} e_{k}\right\|-\left\|f_{k} a f_{k}\right\| \\
& =\left(u\left(\sum_{j=0}^{\infty} \lambda_{j} e_{j}\right) u^{*}\right)_{k, k}-\left\|f_{k} a f_{k}\right\| .
\end{aligned}
$$

Claim 1: $\left(u\left(\sum_{j=0}^{\infty} \lambda_{j} e_{j}\right) u^{*}\right)_{k, k}>1+\left(\lambda_{0}-1\right) / 2^{k+2}$ for all $k$.

For ease of computations, notice that

$$
u_{i, j}= \begin{cases}(1 / \sqrt{2})^{i+1} & j=0 \\ (1 / \sqrt{2})^{i+2-j} & 1 \leq j \leq i \\ -1 / \sqrt{2} & j=i+1 \\ 0 & j>i+1\end{cases}
$$

and thus for all $i, j \geq 0$,

$$
\begin{aligned}
\left(u e_{0} u^{*}\right)_{i, i} & =\left|u_{i, 0}\right|^{2}=1 / 2^{i+1} \\
\left|u_{i, j}\right| & \leq(1 / \sqrt{2})^{i+1-j}
\end{aligned}
$$


Then

$$
\begin{aligned}
\left(u\left(\sum_{j=0}^{\infty} \lambda_{j} e_{j}\right) u^{*}\right)_{k, k} & =\left(u\left(I+\left(\lambda_{0}-1\right) e_{0}+\sum_{j=1}^{\infty}\left(\lambda_{j}-1\right) e_{j}\right) u^{*}\right)_{k, k} \\
& =1+\left(\lambda_{0}-1\right) / 2^{k+1}-\left|\sum_{i=1}^{k+1} u_{k, i}\left(\lambda_{i}-1\right) \overline{u_{k, i}}\right| \\
& \geq 1+\left(\lambda_{0}-1\right) / 2^{k+1}-\sum_{i=1}^{k+1}\left|u_{k, i}\right|^{2}\left|\lambda_{i}-1\right| \\
& >1+\left(\lambda_{0}-1\right) / 2^{k+1}-\sum_{i=1}^{k+1}(1 / 2)^{k+1-i}\left(\lambda_{0}-1\right) / 4^{i+1} \quad \text { (by (1) and }(6) \\
& >1+\left(\lambda_{0}-1\right) / 2^{k+1}-\frac{\left(\lambda_{0}-1\right)}{2^{k+3}} \\
& >1+\left(\lambda_{0}-1\right) / 2^{k+2},
\end{aligned}
$$

which proves Claim 1.

Claim 2: $\left\|f_{k} a f_{k}\right\|<\left(\lambda_{0}-1\right) / 2^{k+2}$ for all $k$.

Indeed,

$$
\begin{aligned}
& \left\|f_{k} a f_{k}\right\|=\left\|\rho\left(u^{*} e_{k} u\right) a \rho\left(u^{*} e_{k} u\right)\right\| \\
& =\left\|q_{k} \rho(u) a \rho(u)^{*} q_{k}\right\| \\
& =\left\|q_{k} \rho(u)\left(\sum_{i=0}^{\infty} q_{i}\right) a\left(\sum_{j=0}^{\infty} q_{j}\right) \rho\left(u^{*}\right) q_{k}\right\| \quad \quad(\text { since } \rho(u)=q \rho(u)=\rho(u) q) \\
& =\left\|\sum_{i, j=0}^{k+1} q_{k} \rho(u) q_{i} a q_{j} \rho\left(u^{*}\right) q_{k}\right\| \\
& \leq \sum_{i, j=0}^{k+1}\left\|q_{k} \rho(u) q_{i}\right\|\left\|q_{j} \rho\left(u^{*}\right) q_{k}\right\|\left\|q_{i} a q_{j}\right\| \\
& =\sum_{i, j=0}^{k+1}\left|u_{k, i}\right|\left|u_{k, j}\right|\left\|q_{i} a q_{j}\right\| \\
& \leq \sum_{i, j=0}^{k+1}\left|u_{k, i}\right|\left|u_{k, j}\right|\left(\left\|q_{i} a_{+} q_{j}\right\|+\left\|q_{i} a_{-} q_{j}\right\|\right) \\
& \leq \sum_{i, j=0}^{k+1}(1 / \sqrt{2})^{k+1-i}(1 / \sqrt{2})^{k+1-j} \frac{\left(\lambda_{0}-1\right)(\sqrt{2}-1)^{2}}{2^{i+j+2}} \\
& =\frac{\left(\lambda_{0}-1\right)(\sqrt{2}-1)^{2}}{2^{k+3}} \sum_{i=0}^{k+1}(1 / \sqrt{2})^{i} \sum_{j=0}^{k+1}(1 / \sqrt{2})^{j} \\
& =\frac{\left(\lambda_{0}-1\right)(\sqrt{2}-1)^{2}}{2^{k+3}} \frac{2}{(\sqrt{2}-1)^{2}}\left(1-\left(\frac{1}{\sqrt{2}}\right)^{k+2}\right)^{2} \\
& <\left(\lambda_{0}-1\right) / 2^{k+2},
\end{aligned}
$$

which proves Claim 2. 
From (4), Claim 1 and Claim 2, we see that for all $k$,

$$
\left\|f_{k} A f_{k}\right\|>1+\left(\lambda_{0}-1\right) / 2^{k+2}-\left(\lambda_{0}-1\right) / 2^{k+2}=1 .
$$

Remark 4.5. The key idea in the proof for Lemma 4.4 above is that by acting within a copy of $\mathbb{B}\left(l_{2}\right)$ that is identified with the corner $q \mathcal{M}(\mathcal{A}) q$ of $\mathcal{M}(\mathcal{A})$, the unitary matrix $u$ permits to turn the diagonal operator $\sum_{k=0}^{\infty} \lambda_{k} q_{k}$ which has one entry larger than 1 and all the other entries "close" to 1 into an operator with all the diagonal entries strictly larger than 1. This "spreading out" action of u can be illustrated directly in $\mathbb{B}\left(l_{2}\right)$ by showing that if for some $t>0$ we set $D:=I+$ te $e_{o} \in$ $\mathbb{B}\left(l_{2}\right)$, i.e., the diagonal operator with diagonal sequence

$$
<1+t, 1,1,1, \cdots>
$$

then the diagonal sequence of $u D u^{*}$ is

$$
<1+1 / 2 t, 1+1 / 2^{2} t, \cdots 1+1 / 2^{n} t, \cdots>
$$

where indeed each diagonal entry is larger than 1.

Proposition 4.6. Let $\mathcal{A}$ be a $\sigma$-unital non-unital purely infinite simple $C^{*}$-algebra and let $A \in \mathcal{M}(\mathcal{A})^{+}$with $\|A\|_{\text {ess }}=1$ and $\|A\|>1$. Then $A$ is a strict sum of projections.

Proof. Since $\|A\|_{e s s}=1$ and $\|A\|>1$, there exists a sequence $\left\{f_{k}\right\}_{k=0}^{\infty}$ of pairwise orthogonal projections in $\mathcal{A}$ satisfying all conditions of Lemma 4.4.

Let $p=\sum_{k=0}^{\infty} f_{k}$. Then $p$ is a projection of $\mathcal{M}(\mathcal{A})$. Rewrite $A$ as

$$
A=A^{1 / 2}(1-p) A^{1 / 2}+\sum_{k=0}^{\infty} A^{1 / 2} f_{k} A^{1 / 2}
$$

where the sum converges in the strict topology in $\mathcal{M}(\mathcal{A})$ and $\left\|A^{1 / 2} f_{k} A^{1 / 2}\right\|>1$ for all $k \geq 0$ by condition (iii) of Lemma 4.4. Now choose a sequence of mutually orthogonal projections $g_{k}$ of $\mathcal{A}$ whose sum converges to the identity. Then $A^{1 / 2}(1-p) g_{k}(1-p) A^{1 / 2} \in \mathcal{A}^{+}$for every $k$ and

$$
A^{1 / 2}(1-p) A^{1 / 2}=\sum_{k=1}^{\infty} A^{1 / 2}(1-p) g_{k}(1-p) A^{1 / 2} .
$$

in the strict topology. Let

$$
a_{k}:=A^{1 / 2}(1-p) g_{k}(1-p) A^{1 / 2}+A^{1 / 2} f_{k} A^{1 / 2} .
$$

Then $a_{k} \in \mathcal{A}^{+},\left\|a_{k}\right\| \geq\left\|A^{1 / 2} f_{k} A^{1 / 2}\right\|>1$ for al $k$ and

$$
A=\sum_{k=1}^{\infty} a_{k}
$$

in the strict topology. By Remark 3.5, $A$ is a strict sum of projections.

This provides the last substantial step in the proof of our main theorem.

Proof of Theorem 1.1. First, the necessity. If $A$ is a strict sum of projections belonging to $\mathcal{A}$, then either the number of projections is finite, in which case $A \in \mathcal{A}$ (case (iv)), or it is infinite, in which case $\|A\|_{\text {ess }} \geq 1$ by Lemma 3.2. It is clear that $\|A\| \geq 1$. If $\|A\|=1$, then $A$ must be itself a projection (case (iii)). Indeed it is 
well known that if $p, q$ are two projections and $\|p+q\|=1$ then $p$ and $q$ must be orthogonal. Finally, if $\|A\|>1$ then we can have either $\|A\|_{\text {ess }}>1$ (case (i)) or $\|A\|_{\text {ess }}=1$ (case(ii).)

Now the sufficiency.

(i) Proposition 3.4

(ii) Proposition 4.6

(iii) If $A=p \in \mathcal{M}(\mathcal{A})$ ) is a projections, then $p \mathcal{A} p$ has an increasing approximate identity of projections ([28]), say $f_{n}$, and hence, $A=p=\sum_{n=1}^{\infty}\left(f_{n}-f_{n-1}\right)$ as a strict sum of projections of $\mathcal{A}$ (where $f_{0}$ ).

(iv) Nothing to prove.

Remark 4.7. We would like to point out that due to [12, Theorem 1.1], the operator $A=I+(1 / 2) e \in \mathbb{B}\left(l_{2}\right)$, where $e$ is a rank one projection, cannot be written as a strongly convergent sum of projections in $\mathbb{B}\left(l_{2}\right)$. However, if we unitarily embed $\mathbb{B}\left(l_{2}\right)$ in the multiplier algebra $\mathcal{M}(\mathcal{A})$ where $\mathcal{A}$ is a $\sigma$-unital, nonunital purely infinite simple $C^{*}$-algebra, then $A$ can be written as a strictly convergent sum of projections in $\mathcal{A}$. This is due to the much richer structure of $\mathcal{M}(\mathcal{A})$ than $\mathbb{B}\left(l_{2}\right)$.

\section{REFERENCES}

[1] J. Antezana, P. Massey, M. Ruiz and D. Stojanoff, The Schur-Horn Theorem for operators and frames with prescribed norms and frame operator Illinois J. of Math., (2007) Preprint

[2] B. Blackadar, K-theory for operator algebras, (1986) Springer-Verlag, New York

[3] L. Brown and G. Pedersen, $C^{*}$-algebras of real rank zero J. Funct. Anal., 99 (1991) 131-149

[4] K. Davidson, $C^{*}$-algebras by example Fields Institute Monographs, 6 (1996) American Mathematical Society, Providence, RI

[5] K. Dykema, D. Freeman, K Kornelson, D. Larson, M. Ordower and E. Weber, Ellipsoidal tight frames and projection decompositions of operators Illinois J. Math., 48 (2004) no. 2, 477-489

[6] G. A. Elliott, Derivations of matroid $C^{*}$-algebras. II. Ann. of Math. (2), 100 (1974) 407-422

[7] G. A. Elliott and G. Gong, On the classification of $C^{*}$-algebras of real rank zero. II Ann. of Math. (2), 144 (1996) no. 3, 497-610

[8] G. Elliott and M. Rordam, Perturbation of Hausdorff moment sequences, and an application to the theory of $C^{*}$-algebras of real rank zero Operator Algebras, The Abel Symposium 2004, (2006) Springer Verlag, 97-115

[9] P. Fillmore, On sums of projections J. Funct. Anal., 4 (1969) 146-152

[10] M. Frank and D. Larson, A module frame concept for Hilbert $C^{*}$-modules Contemp. Math., 247 (1999) 207-233

[11] M. Frank and D. Larson, Frames in Hilbert $C^{*}$-modules and $C^{*}$-algebras J. Operator Theory, 48 (2002) no. 2, 273-314

[12] V. Kaftal and P. W. Ng and S. Zhang, Strong sums of projections in $\mathbb{B}(\mathcal{H})$ and in von Neumann factors J. Funct. Anal, 257 (2009) 2497-2529

[13] K. Kornelson and D. Larson, Rank-one decomposition of operators and construction of frames Contemp. Math., 345 (2004) 203-214

[14] H. Lin, Skeleton $C^{*}$-algebras Canad. J. Math., 44 (1992) 324-341

[15] H. Lin, Generalized Weyl-von Neumann theorems. II. Math. Scand, 77 (1995) no. 1, 129-147

[16] H. Lin, Embedding an AH-algebra into a simple $C^{*}$-algebra with prescribed $K K$-data $K$ Theory, 24 (2001) no. 2, 135-156

[17] H. Lin and S. Zhang, On infinite simple $C^{*}$-algebras J. Funct. Anal., 100 (1991) no. 1, 221-231 
[18] H. Lin, An introduction to the classification of amenable $C^{*}$-algebras World Scientific Publishing Co., Inc., (2001) River Edge, NJ

[19] F. Perera and M. Rordam, $A F$-embeddings into $C^{*}$-algebras of real rank zero J. Funct. Anal., 217 (2004) no. 1, 142-170

[20] N.E. Wegge-Olsen, K-Theory and $C^{*}$-Algebras Oxford University Press, Oxford, (1993)

[21] S. Zhang, On the structure of projections and ideals of corona algebras Canad. J. Math., 41 (1989) no. 4, 721-742

[22] S. Zhang, A property of purely infinite simple $C^{*}$-algebras Proc. Amer. Math. Soc., 109 (1990) no. 3, 717-720

[23] S. Zhang, $C^{*}$-algebras with real rank zero and the internal structure of their corona and multiplier algebras. III Canad. J. Math., 42 (1990) no. 1, 159-190

[24] S. Zhang, Diagonalizing projections in multiplier algebras and in matrices over a $C^{*}$-algebra Pacific J. Math, 145 (1990) no. 1, 181-200

[25] S. Zhang, A Riesz decomposition property and ideal structure of multiplier algebras J. Operator Theory, 24 (1990) no. 2, 209-225

[26] S. Zhang, Matricial structure and homotopy type of simple $C^{*}$-algebras with real rank zero J. Operator Theory, 26 (1991) 283-312

[27] S. Zhang, Ideals of generalized Calkin algebras Contemp. Math., 120 (1991) 193-198

[28] S. Zhang, K1-groups, quasidiagonality, and interpolation by multiplier projections Trans. Amer. Math. Soc., 325 (1991) no. 2, 793-818

[29] S. Zhang, Certain $C^{*}$-algebras with real rank zero and their corona and multiplier algebras. I. Pacific J. Math., 155 (1992) no. 1, 169-197

[30] S. Zhang, $C^{*}$-algebras with real rank zero and their corona and multiplier algebras. IV Internat. J. Math., 3 (1992) no. 2, 309-330

[31] S. Zhang, Certain $C^{*}$-algebras with real rank zero and their corona and multiplier algebras. I Pacific J. Math., 155 (1992) no. 1, 169-197

[32] S. Zhang, Certain $C^{*}$-algebras with real rank zero and their corona and multiplier algebras. II $K$-theory, 6 (1992) no. 1, 1-27

[33] S. Zhang, Quasidiagonalizing unitaries and the generalized Weyl-von Neumann theorem Algebraic methods in operator theory, (1994) Birkhauser, Boston, MA, USA

Department of Mathematics, University of Cincinnati, P. O. Box 210025, Cincinnati, $\mathrm{OH}, 45221-0025$, USA

E-mail address: victor.kaftal@UC.Edu

Department of Mathematics, University of Louisiana, 217 Maxim D. Doucet Hall, P.O. Box 41010, Lafayette, Louisiana, 70504-1010, USA

E-mail address: png@louisiana.edu

Department of Mathematics, University of Cincinnati, P.O. Box 210025, Cincinnati, $\mathrm{OH}$, 45221-0025, USA

E-mail address: zhangs@email.uc.edu 